Ann. Génét. Sél. anim., I973, 5 (I), 39-5I.

\title{
DIFFÉRENCES DE MORTALITÉ EMBRYONNAIRE OU POST-EMBRYONNAIRE ASSOCIÉES A CERTAINS GÈNES CONNUS CHEZ LA POULE (1)
}

\author{
P. MÉRAT \\ avec la collaboration technique de G. Coquerelle \\ Laboratoire de Génétique factorielle, \\ Centre national de Recherches zootechniques, I. N. R. A. \\ 78350 Jouy en Josas
}

\section{RÉSUMÉ}

Nous avons cherché des associations possibles entre certains gènes connus à effet visible (coloration ou morphologie), dans des familles en ségrégation pour ces gènes, et le taux de mortalité à trois stades (en fin d'incubation, chez les poussins jusqu'à l'âge de 8 semaines, chez les poulettes de 8 semaines à ro mois). Les principaux résultats sont les suivants :

Pour trois gènes de coloration $(C, I, E)$, les embryons plus mélanisés ont une mortalité de I, 5 à 3 p. roo supérieure en fin d'incubation; cette différence se prolonge dans les premières semaines de la vie du poussin pour les gènes $C$ et peut-être $I$.

Au locus $K$ se manifeste un excès (+ 3 p. roo) du pourcentage de morts en fin d'incubation parmi les embryons à emplumement précoce comparés à ceux à emplumement tardif.

Les embryons et les poussins à crête en pois $(P p)$ ont une survie meilleure $(+5 \mathrm{et}+2 \mathrm{p}$. 1oo respectivement aux deux stades) que ceux à crête simple $(p p)$.

La mortalité des poussins à 5 doigts $(P o p o$ ou $P O P O)$ est d'environ $2 \mathrm{p}$. roo plus élevée que celle des poussins à 4 doigts, l'inverse existant peut-être avant l'éclosion.

Pour les poulettes après 8 semaines, la seule différence significative concerne le locus $B l$ : Les +9 hétérozygotes pour la dilution du noir dans le plumage $(B l b l)$ ont une mortalité de 5 p. Ioo inférieure à celle des homozygotes à noir non dilué $b l b l$.

Aux divers stades, un taux de survie moins bon des homozygotes à crête en rose $R R$ comparés aux autres génotypes est suggéré, mais demande confirmation par des données plus nombreuses.

La mortalité en fin d'incubation est plus grande $(+3 \mathrm{p}$. Ioo) lorsque la mère est colorée $(C c)$ que lorsqu'elle est homozygote pour le "blanc récessif " $(c c)$. Par contre, la mortalité juvénile et peut-être embryonnaire est plus faible lorsque la mère est "dorée " $\left(s^{-}\right)$que lorsqu'elle est " argentée " $\left(S^{-}\right)$.

Après 8 semaines, les poulettes issues d'un croisement entre une mère hétérozygote pour le gène $E$ (extension du noir), et un mâle ne possédant pas cet allèle, paraissent avoir une mortalité plus faible que celles provenant du croisement réciproque.

L'intérêt de ces résultats, au cas où ils seraient applicables à d'autres populations, est brièvement discuté.

(1) Ce travail a été réalisé en partie grâce à une subvention de l'Institut technique de l'Aviculture (I. T. A. V. I.), 28, rue du Rocher, Paris $(8 \mathrm{e})$. 


\section{INTRODUCTION}

A l'exception des gènes contrôlant le groupe sanguin ou des polymorphismes biochimiques, peu de résultats ont été publiés concernant la possibilité d'associations entre des gènes connus et la mortalité embryonnaire. HuTT (I95I) a noté l'existence d'un taux d'éclosion inférieur pour les poussins porteurs de l'allèle $E$ (extension du noir) : Il attribue ce fait à leur duvet plus court, qui gênerait les mouvements de rotation à 1'intérieur de la coquille avant l'éclosion. Selon BERNIER et COONEY (I954), les besoins en riboflavine de ces mêmes poussins sont augmentés.

D'après nos propres résultats (MÉRAT, I967 a), la mortalité des embryons colorés $(C c)$ à la fin de la période d'incubation apparaît plus élevée que celle des embryons à duvet blanc $(c c)$.

Selon Gredina (citée par LANDAUER, I967), le taux d'éclosion pourrait être meilleur pour les embryons à emplumement rapide (allèle $k$ ) que pour ceux à emplumement lent (allèle $K$ ).

Pour la mortalité post-embryonnaire, BRUMBaUgh et Hol,LANDER (I966) suggèrent une différence liée à des gènes d'extension du noir. WASHBURN et SMYTH (I958) attribuent des cas de mortalité par anémie à un gène lié au facteur $C o$ responsable du plumage " herminé ".

Nous avons voulu, dans le présent travail, chercher d'une façon plus générale les associations possibles d'un certain nombre de gènes connus à effet extérieur (coloration ou morphologie) avec le taux de mortalité, pendant l'incubation ou ultérieurement.

\section{MATÉRIEL ET MÉTHODES}

Des articles antérieurs (MÉRAT, I962, I967 a) apportent des précisions sur la population étudiée, où un certain nombre de gènes sont maintenus en ségrégation.

Dans ce troupeau, le nombre d'œufs "clairs " et d'embryons morts à $\mathbf{I} 8$ jours était relevé dans chaque famille de frères-sœurs. Certains gènes à effet visible étaient identifiés, d'après le phénotype, chez des embryons morts après le $\mathrm{I} 8 \mathrm{e}^{\mathrm{e}}$ jour d'incubation, depuis $\mathrm{I} 959$ inclus. Le sexe de ces embryons était reconnu par observation directe des gonades lorsque leur état le permettait. A l'éclosion, le phénotype de tous les poussins était décrit pour chaque gène. Il était ensuite confirmé à l'âge de 8 semaines, et le sexe enregistré.

Après l'éclosion, la mortalité individuelle était enregistrée. Nous la classons en deux périodes successives : avant 8 semaines (élevage au sol pour tous les poussins nés viables, les deux sexes étant réunis jusqu'en 1968 , séparés depuis); de 8 semaines à l'arrêt du contrôle de la ponte, vers Io mois d'âge, pour les femelles gardées après 8 semaines (à cet âge, seules les familles les plus nombreuses étaient conservées). Étant donné les effectifs à notre disposition et l'impossibilité d'un diagnostic précis pour chaque cas, nous considérons seulement la mortalité globale non accidentelle pour chacun de ces stades.

Les loci pour lesquels nous avons identifié les génotypes d'après l'apparence de l'embryon entre le $18 \mathrm{e}$ et le $2 \mathrm{I}^{\mathrm{e}}$ jour sont indiqués dans la première partie du tableau $\mathrm{I}$.

Pour ces gènes, la reconnaissance des phénotypes figurant au tableau i était, la plupart du temps, sans ambiguïté même avant l'éclosion. L'absence de pigmentation du duvet associée au blanc récessif ou dominant se distinguait d'autant mieux de la présence de pigmentation que, dans la majorité des cas correspondants, les animaux possédaient le génotype $s s$ ou $s^{-}$permettant l'expression des mélanines rouges. De toutes façons, la présence ou l'absence d'une coloration noire des rémiges était le plus souvent un critère suffisant. Quant au gène $S$, lorsqu'il était présent, il se reconnaissait précisément par la suppression des phæomélanines du duvet. Les gènes $R$ et $P$ 
de forme de la crête et le gène $\mathrm{Na}$ ("Cou nu ") ne comportaient pratiquement jamais de difficultés d'identification. Enfin, les allèles $K$ et $k$ (emplumement tardif ou précoce) se reconnaissent déjà, apparemment, au $\mathrm{I} 8^{\mathrm{e}}$ jour par le même effet qu'à l'éclosion sur la longueur relative des rémiges et des couvertures.

Pour le gène d'extension du noir, nous n'avons pas tenté de faire une distinction entre différents allèles possibles de $E$, que nous désignons arbitrairement par " $e$ ". Quant à la polydactylie, nous indiquons seulement le phénotype ( 5 ou 4 doigts), la pénétrance du gène $P o$ n'étant pas toujours totale.

En outre, après 8 semaines d'âge, la mortalité des $\$+q$ était comparée pour les génotypes ou phénotypes correspondant à d'autres loci $\left(2^{\mathrm{e}}\right.$ partie du tableau $\left.\mathrm{r}\right)$. Pour ces loci, quelques difficultés d'identification existaient. Pour le facteur "herminé " ou "sauvage " $(C o)$, le génotype enregistré est seulement le plus vraisemblable. Aux loci $B$ et $B l$, une fraction (relativement réduite) des femelles respectivement $B^{-}$ou $B l b l$ n'a probablement pas été reconnue; d'où un certain déficit des catégories correspondantes.

TABLEAU I

Phénotypes reconnus à divers stades

I. - Depuis le stade " embryon de 18 jours"

\begin{tabular}{|c|c|}
\hline Locus & Phénotypes identifiés et génotypes correspondants dans la population \\
\hline$C$ & Présence de mélanines dans le duvet $(C C$ ou $C c)$ - absence $(c c)$ \\
\hline$I$ & Présence de mélanines noires $(i i)$ - absence $(I i)$ \\
\hline$E$ & Duvet à noir étendu $(E e)-$ noir restreint $(e e)$ \\
\hline$S$ & Absence de mélanines rouges ( $S s$ ou $\left.S^{-}\right)$- présence ( $s s$ ou $s^{-}$) \\
\hline$K$ & Emplumement précoce $\left(k k\right.$ ou $\left.k^{-}\right)-\operatorname{tardif}\left(K k\right.$ ou $\left.k^{-}\right)$ \\
\hline$R$ & Crête en rose $(R R$ ou $R r)$ - crête simple $(r r)$ \\
\hline$P$ & Crête en pois $(P p)$ - crête simple $(p p)$ \\
\hline $\mathrm{Na}$ & Duvet " cou nu" $(\mathrm{NaNa})-$ normal (nana) \\
\hline Po & Polydactylie (PoPo ou Popo) - normal ( $p \circ p o)$. \\
\hline
\end{tabular}

* $e$ représentant ici tout allèle autre que $\mathrm{E}$.

II. - A partir de 8 semaines d'âge

$B \quad$ Plumage «barré " $\left(B^{-}\right)-$non barré $\left(b^{-}\right)$

$B l \quad$ Mélanines noires diluées dans le plumage $(B l b l) \rightarrow$ non diluées $(b l b l)$

Co Plumage à noir restreint type "herminé " (Coco) — " type sauvage " (caco)

$W \quad$ Présence de xanthophylles épidermiques $(w w)-$ absence $(W W$ ou $W w)$

Les années étudiées allaient de I959 à I970 inclus pour la mortalité embryonnaire, et de I955 à I970 pour la mortalité post-embryonnaire.

La comparaison de la mortalité par génotypes pour un locus et un stade donnés était limitée aux familles où apparaissait, au point de départ du stade en question, une ségrégation mendélienne pour ce locus.

En règle générale, les génotypes comparés étaient l'hétérozygote et l'homozygote récessif, issus de croisements du type $\delta^{\star} A a \times$ 우 $a a$ ou $\delta^{\star} a a \times q A a$. Faisaient exception, pour la mortalité des embryons et des jeunes, les loci $C$ et $R$, où respectivement les croisements $C c \times C c$ et $R r \times R r$ étaient représentés par des effectifs relativement importants. Nous indiquons séparément les résultats relatifs à ces croisements. Quant au locus $E$, nous n'avons pas fait cette sépa- 
ration, la distinction phénotypique entre $E E$ et $E e$ n'ayant pas toujours été clairement faite.

Pour chaque stade et chaque locus, le taux de mortalité des différents génotypes était comparé par un test de $\chi^{2}$ sur le résultat global de l'ensemble des années. Un $\chi^{2}$ est calculé de façon analogue pour comparer la mortalité dans des croisements réciproques relatifs à certains loci.

Le fait que la probabilité de survie ne soit pas indépendante dans une même famille et une même génération ne doit pratiquement pas diminuer le degré de signification du premier test (comparaison des génotypes individuels) : Comme nos données n'incluent que des familles contenant à la fois les deux génotypes comparés, les écarts au taux de mortalité moyen attachés à une famille se retrouvent a priori dans ces deux génotypes et ne peuvent causer une différence appréciable dans leur pourcentage de mortalité. Par contre, ceci peut jouer un rôle non entièrement négligeable dans la comparaison des croisements réciproques, si le nombre de familles qui figurent dans chacun n'est pas très considérable, et la signification des $\chi^{2}$ que nous présentons dans ce cas peut n'être qu'approximative.

Les effectifs présentés dans l'analyse de la mortalité embryonnaire et dans celles de la mortalité des jeunes ne se correspondent pas, le phénotype des embryons morts n'ayant pas été relevé les premières années. D'autre part, dans les croisements du type $A a \times A a$, il y a en général un certain excès du phénotype récessif : ceci s'explique, du fait que les familles choisies comme représentatives de ce type de croisement étaient celles qui présentaient une ségrégation; d'où un léger "biais " augmentant la proportion apparente d'homozygotes récessifs.

\section{RÉSULTATS}

\section{A. - Différences entre zygotes de génotype différent}

$\mathrm{I}^{0}$ Le résultat des comparaisons relatives à la mortalité en fin d'incubation (182I jours) et chez le poulet de l'éclosion à l'âge de 8 semaines est contenu dans le tableau 2.

Aux loci " $C$ ", " $I$ » et " $E$ ", les embryons plus mélanisés ont une mortalité légèrement supérieure en fin d'incubation : respectivement $+\mathrm{I}, 3 ;+2,0$ et $+2,8 \mathrm{p}$. Ioo. Cette différence est hautement significative dans les trois cas, et parait se prolonger dans les premières semaines de la vie du poussin pour les gènes $C$ et peut-être $I$. Elle ne peut s'expliquer par le classement erroné d'une certaine proportion d'embryons du génotype le moins pigmenté dans la catégorie la plus pigmentée. Nous avons déjà indiqué que l'identification des génotypes à ces trois loci était en règle générale sans ambiguité. De plus, les erreurs possibles seraient plutôt en sens inverse, consistant à noter " blancs » des embryons $C c$ ou respectivement $i i$ dont la pigmentation des rémiges serait peu étendue.

Quelques résultats relatifs au croisement $C c \times C c$ n'indiquent pas, à première vue, un effet différent associé aux génotypes $C C$ et $C c$, si on les compare à ceux des croisements $C c \times c c$ ou $c c \times C c$.

De même, au locus " $K$ ), 1'excès $(+2,8$ p. Ioo) très hautement significatif du pourcentage d'embryons morts en fin d'incubation et notés " emplumement précoce " comparés à ceux à " emplumement tardif " serait très difficilement explicable par la classification erronée d'embryons de génotype $K k$ ou $K$ - en catégorie " précoce "; on s'attendrait plutôt, a priori, à voir des embryons $k k$ ou $k$ - classés "tardifs" à ce stade si leurs rémiges n'avaient pas atteint la taille qu'elles ont à l'éclosion. De toutes façons, nous avions ici un moyen de contrôle grâce au linkage entre les gènes $S$ et $K$. Dans une fraction appréciable des données, l'allèle $K$ était lié à $S$ et $k$ à $s$. En ne prenant en considération comme embryons ou poussins à emplumement tardif que ceux marqués par le gène $S$, et comme zygotes à emplumement précoce que 
GÈNES ET MOR'TALITÉ CHEZ IA POULE

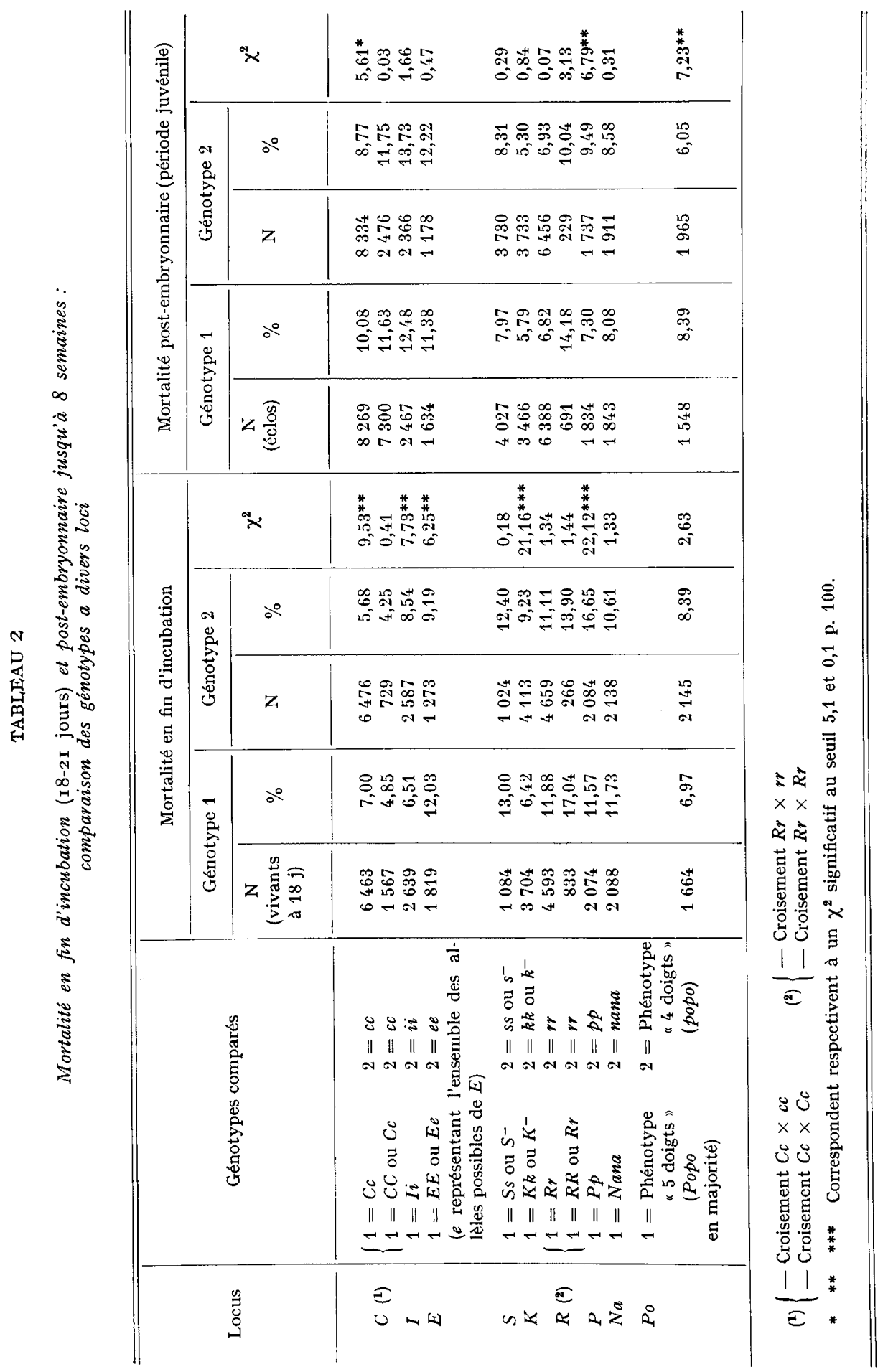


ceux marqués par $s$, on observe, sur 272 I zygotes de la première catégorie, I34 embryons morts du I8 e au $2 I^{\mathrm{e}}$ jour (4,9 p. IOO) et $\mathrm{I} 86$ morts sur 2995 zygotes du second groupe, soit 6,2 p. Ioo ( $\left.{ }^{1}\right)$. La différence est significative au seuil 5 p. Ioo $\left(\chi^{2}=4,43\right)$. Comme, par ailleurs, la comparaison globale directe entre embryons porteurs de $S$ ou de $s$ n'indique aucune différence décelable de mortalité au même stade, ceci nous paraît confirmer l'effet associé au gène $K$, concernant cette mortalité.

Pour le gène " $P$ ", les embryons et les poussins à phénotype "crête en pois » ont une survie nettement meilleure que ceux à crête simple. Ici également, il est douteux que la différence observée à la phase embryonnaire puisse s'expliquer par la nonreconnaissance d'une petite fraction des embryons de génotype $P p$ et leur classement dans la catégorie "crête simple " car, comme nous l'avons noté plus haut, la différence entre les deux phénotypes, dès cet âge, apparaît bien tranchée. Ceci n'expliquerait pas, d'ailleurs, la différence de survie notée après l'éclosion où les vérifications à un âge ultérieur et les tests de descendance permettent de vérifier que les erreurs sont exceptionnelles.

Toutefois, certains embryons concernés ici étaient d'autre part du génotype $R r$, et l'on pouvait davantage penser, parmi eux, à une difficulté de la reconnaissance des génotypes au locus $P$, entre crêtes " en noix " $(P p)$ ou " en rose " $(p p)$. Cependant, en retranchant ces embryons, les nombres respectifs d'embryons morts dans les catégories "crête en pois " et " crête simple " sont I94 (sur 2028 vivants à I8 jours, soit 9,56 p. Ioo) et 26 I (sur I 998, soit I3,06 p. I00). La différence est encore significative au seuil I p. Ioo $\left(\chi^{2}=I 2,28\right)$. La conclusion reste donc la même.

Au locus " $P o$ ", la mortalité juvénile plus élevée des poussins à 5 doigts semblerait, à première vue, compenser une différence en sens inverse existant en fin d'incubation.

D'une façon générale, enfin, dans aucun des cas où une différence significative de mortalité était observée entre génotypes, nous n'avons trouvé de différence de mortalité entre sexes à l'intérieur de chaque génotype, tant au stade embryonnaire qu'au stade poussin.

$2^{\circ}$ Les comparaison relatives à la mortalité des poulettes après 8 semaines d'âge sont présentées au tableau 3. A ce stade, n'ayant pas constaté en général de différence entre génotype homozygote dominant et hétérozygote, nous présentons tous les croisements regroupés, y compris cєux du type $A a \times A a$. Une exception est faite pour le locus $R$, pour lequel les résultats sont détaillés plus loin.

La seule différence significative concerne ici le gène " $B l$ ". Les poulettes "blue " hétérozygotes $(B l b l)$ ont une mortalité inférieure à celle d€s homozygotes à noir non dilué $(b l b l)$, de façon très hautement significative $(P<0,00 \mathrm{I})$. Cette différence se retrouve de même sens dans la majorité des années étudiées ( 77 générations, de printemps ou d'automne, sur 23) avec une faible différence non significative en sens contraire pour les six autres ; et ro fois sur I 2 générations ayant des effectifs suffisants pour le calcul d'un $\chi^{2}$ de contingence. Nous avions déjà signalé une tendance dans ce sens (MÉrat, I962). Les quelques années qui ont suivi ne la confirmaient pas de

(1) On compte d'autre part I $_{5}$ embryons morts de phénotype $(s k)$ et $34(S k)$ contre respectivement 40 et $57 \mathrm{chez}$ les survivants. Le taux de recombinaison apparent est voisin de I $3 \mathrm{p}$. Ioo chez les morts contre moins de 2 p. roo chez ces derniers. Nous pensons que ceci provient d'erreurs dans l'identification de duvets " argentés " ou "dorés " chez l'embryon. Même compte tenu de ces "recombinés ", la conclusion est la même pour la survie, comparée suivant l'emplumement. 
façon nette (MÉRAT, I970). En fin de compte, nos données actuelles, plus complètes, la révèlent de nouveau.

On vérifie que la probabilité, parmi les I6 gènes étudiés, d'en obtenir un qui donne par hasard un $\chi^{2}$ au moins égal à II, 56 est faible (inférieure à 0,005 ).

Sur les pourcentages par génotype et par année transformés en angles, en enlevant les années de faible effectif, une comparaison du taux de mortalité des deux génotypes $B l b l$ et $b l b l$ par la méthoda des couples, sur la base des différences de

\section{TABLEAU 3}

Mortalité des poulettes de 8 semaines à 10 mois d'âge: comparaison des génotypes a divers loci

\begin{tabular}{|c|c|c|c|c|c|c|c|}
\hline \multirow[b]{2}{*}{ Locus } & \multirow{2}{*}{\multicolumn{2}{|c|}{ Génotypes comparés }} & \multicolumn{2}{|c|}{ Génotype 1} & \multicolumn{2}{|c|}{ Génotype 2} & \multirow[b]{2}{*}{$\chi^{2}$} \\
\hline & & & $\begin{array}{c}\mathrm{N} \\
\text { (vivantes } \\
\text { à } 8 \text { sem.) }\end{array}$ & p. 100 & $\mathrm{~N}$ & p. 100 & \\
\hline$C$ & $1=C C$ ou $C c$ & $2=c c$ & 4398 & 14,62 & 2739 & 14,96 & 0,17 \\
\hline$I$ & $1=I i$ & $2=i i$ & 830 & 24,94 & 826 & 22,52 & 1,34 \\
\hline$E$ & $1=E E$ ou $E e\left(^{(1)}\right.$ & $2=e e$ & 673 & 24,37 & 847 & 25,50 & 0,26 \\
\hline$S$ & $1=S^{-}$ & $2=s^{-}$ & 2076 & 25,72 & 1991 & 24,56 & 0,73 \\
\hline Co & $\begin{array}{c}1=\text { Phénotype } \\
\text { "colombien" } \\
\text { (en principe } \\
\text { Coco) }\end{array}$ & $\begin{array}{c}2=\text { phénotype } \\
\text { sauvage } \\
\text { (en principe } \\
\text { coco) }\end{array}$ & 6146 & 20,01 & 5309 & 20,02 & 0,00 \\
\hline$B$ & $1=B^{-}$ & $2=b^{-}$ & 1039 & 23,58 & 1340 & 21,94 & 0,90 \\
\hline$B l$ & $1=B l b l$ & $2=b l b l$ & 1240 & 13,79 & 1497 & 18,84 & $12,49^{* * *}$ \\
\hline$W$ & $1=W W$ ou $W w$ & $2=w w$ & 4709 & 20,00 & 3198 & 18,82 & 1,69 \\
\hline & $1=K^{-}$ & $2=k^{-}$ & 2013 & 20,36 & 2062 & 21,29 & 0,53 \\
\hline$\left({ }^{2}\right)$ & $1=R r$ & $2=r r$ & 2632 & 17,36 & 2566 & 16,99 & 0,13 \\
\hline & $1=R R$ ou $R r$ & $2=r r$ & 300 & 22,33 & 96 & 23,95 & 0,11 \\
\hline$P$ & $1=P p$ & $2=p p$ & 537 & 10,24 & 490 & 10,61 & 0,04 \\
\hline $\mathrm{Na}$ & $1=N a n a$ & $2=n a n a$ & 358 & 17,32 & 426 & 22,30 & 3,01 \\
\hline Po & $\begin{aligned} 1= & \text { phénotype } \\
& 5 \text { doigts } \\
& \text { (en majorité } \\
& P o p o)\end{aligned}$ & $\begin{array}{c}2=\underset{4 \text { phénotype }}{4 \text { doigts }} \\
(\text { popo })\end{array}$ & 401 & 19,70 & 423 & 19,89 & 0,01 \\
\hline$M b$ & $1=M b m b$ & $2=m b m b$ & 245 & 17,14 & 204 & 13,24 & 1,31 \\
\hline $\mathrm{Cr}$ & $1=C r c r$ & $2=c r c r$ & 181 & 23,12 & 160 & 21,25 & 0,03 \\
\hline
\end{tabular}

(i) $e$ désignant l'ensemble des allèles possibles de $E$.

(2) $\left\{\begin{array}{l}\text { - Croisement } R r \times r r \\ \text { - Croisement } R r \times R r\end{array}\right.$

chaque année, donne $t=2,25$ pour I6 1. d., d'où $P<0,05$. Quoique les pourcentages dans les différentes années retenues soient basés sur des effectifs inégaux, rendant ce test approximatif, ceci paraît renforcer la conclusion qui précède.

Il ne semble pas que la différence du taux de mortalité observée s'accompagne d'une différence de l'âge moyen de mortalité. 


\section{Cas $d u$ locus $\mathrm{R}$.}

D'autre part, dans la descendance du croisement $R r \times R r$, le taux de mortalité est respectivement égal à 22,3 p. 100 (sur 300 vivantes à 8 semaines) et 24,0 p. 100 (sur 96) chez les filles à crête en rose et chez celles à crête simple; les chiffres correspondants sont I4,I p. Ioo (sur 736) et I7,4 p. Ioo (sur 706) pour les croisements $R r \times r r$ et $r r \times R r$ réunis, la comparaison étant limitée aux Io générations où existent simultanément ces divers types de croisement. La différence de mortalité entre le croisement $R r \times R r$ et les autres apparaît significative chez les filles à crête en rose $\left(\chi^{2}=\right.$ Io,43 d'où $\left.P<0,001\right)$ et ne l'est pas chez celles à crête simple $\left(\chi^{2}=2,40\right)$. La valeur du premier $\chi^{2}$ suggère l'existence d'une différence, même si le degré de signification est surestimé du fait de la non-indépendance des données individuelles, groupées par familles. Or, les descendants à crête en rose sont tous de génotype $R r$ pour le deuxième groupe de croisements, mais ceux issus du premier croisement sont $R R$ dans la proportion théorique d'un tiers. D'autre part, nous avons déjà noté l'absence de différence entre les croisements $R r \times r r$ et $r r \times R r$, indiquant l'absence d'effet maternel associé au génotype $R r$. On pourrait donc penser à une mortalité plus grande des poulettes $R R$. Par contre, à l'intérieur du croisement $R r \times R r$, la mortalité des zygotes de phénotype "crête en rose " ou "crête simple " (ces derniers d'effectif peu considérable il est vrai) ne diffère pas significativement. Il est donc difficile de conclure, et des résultats supplémentaires sont nécessaires.

La comparaison des mêmes croisements, pour la mortalité en éclosoir et au stade poussin, ne présente pas de différence significative. D'autre part, à l'intérieur du croisement $R r \times R r$, la mortalité des zygotes à crête en rose est légèrement plus élevée que celle des zygotes à crête simple : Au stade embryonnaire, I7,04 p. Ioo sur 833 vivants à 18 jours pour les premiers, I3,90 p. Ioo sur 266 pour les seconds; pour les poussins, I4,I8 p. IOO sur $6 \mathrm{gr}$ éclos à crête en rose contre Io,04 p. IoO sur 229 éclos à crête simple. La différence de pourcentage n'est pas significative à chaque stade $\left(\chi^{2}=1,44\right.$ et 2,57 respectivement) ; elle suggère encore, cependant, un effet légèrement défavorable associé au génotype $R R$.

\section{B. - Différences entre croisements réciproques}

\section{Mortalité en fin d'incubation et au stade poussin.}

Le tableau 4 indique, à ces stades, la mortalité comparée des croisements réci-

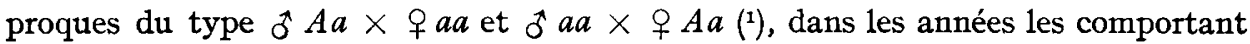
tous deux, pour les loci figurant au tableau 2, à l'exception de $P o$, où les effectifs utilisables étaient insuffisants.

Au locus $C$, en fin d'incubation, la mortalité est plus grande (d'environ 3 p. Ioo) lorsque la mère est du génotype $C c:$ La valeur extrêmement élevée du $\chi^{2}$ apparait suffisamment probante, même avec la réserve déjà formulée par ailleurs de l'existence d'une corrélation entre résultats individuels intra-famille. Au locus $R$, une éclosion légèrement moins bonne est suggérée lorsque la mère est $R r$; de même au locus $S$ pour la mortalité des jeunes lorsque la mère est $S$.

(1) Ou des croisements dont le génotype de la mère diffère, pour les gènes liés au sexe $S$ et $K$. 

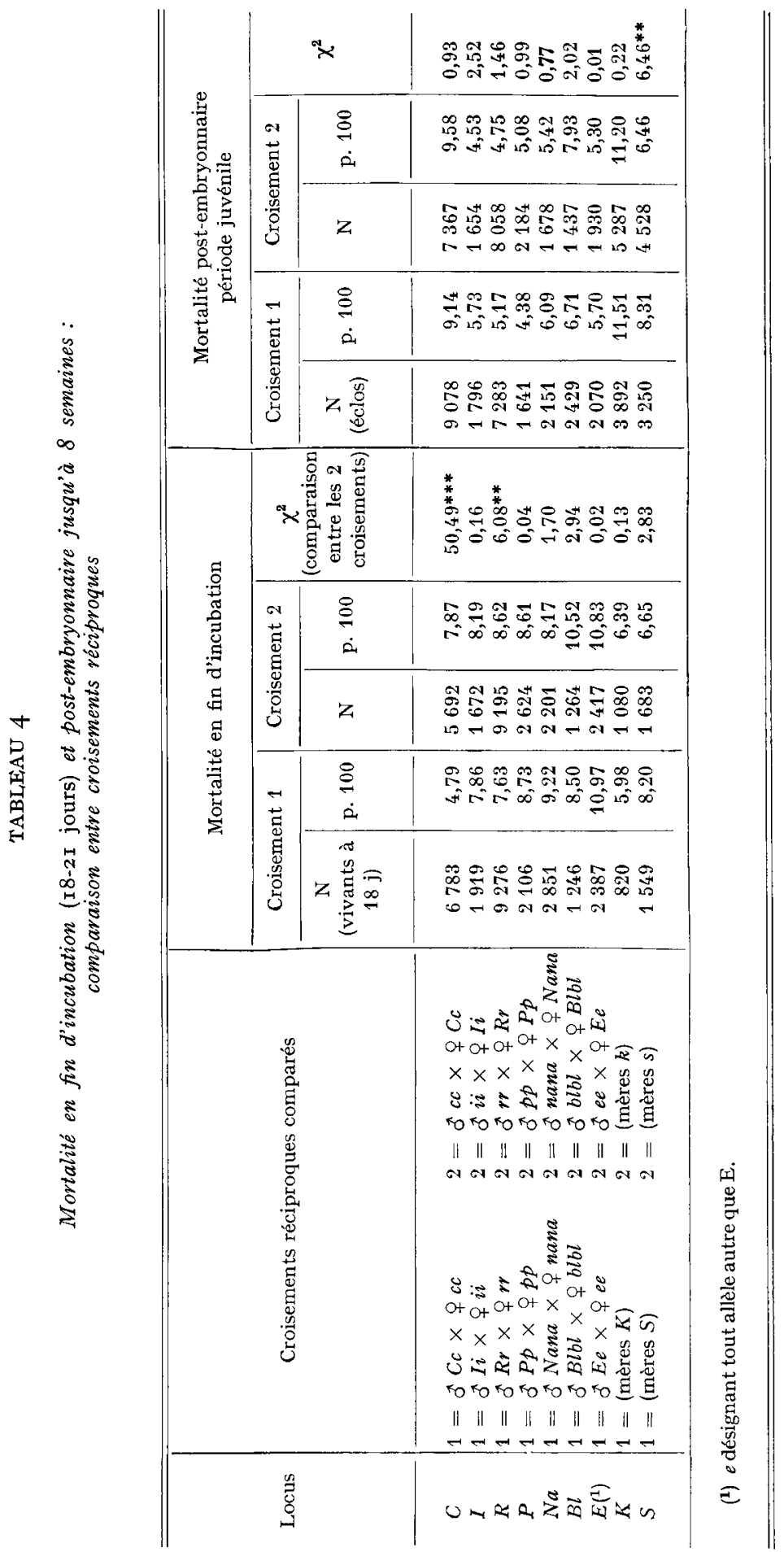
2. Mortalité des poules après 8 semaines.

Seule une différence entre croisements réciproques au locus $E$ se révélant significative, nous ne présentons pas le détail des autres.

$\mathrm{A}$ ce locus, lorsque la mère ne possède aucun allèle $E$, le père étant hétérozygote pour $E$, sur 797 femelles vivantes à 8 semaines, I6,43 p. Ioo sont mortes, sur l'ensemble des années, entre 8 semaines et II mois d'âge. Par contre, les mêmes années, lorsque la mère est hétérozygote pour $E$, sur 772 poulettes vivantes à 8 semaines, 9,84 p. Ioo sont mortes pendant la même période.

La différence entre les deux croisements réciproques est significative au seuil I pour mille $\left(\chi^{2}=14,94\right)$, et elle se retrouve de même sens dans la majorité des générations étudiées.

\section{DISCUSSION ETT CONCLUSIONS}

\section{A. - Différences entre zygotes}

En ce qui concerne la mortalité embryonnaire tardive, nous avons fait remarquer un parallélisme entre les résultats relatifs à plusieurs gènes de pigmentation, les embryons plus pigmentés ayant, dans l'ensemble, une mortalité un peu plus grande. HưT (I95I) avait observé un taux d'éclosion inférieur pour les embryons à duvet noir étendu (allèle $E$ ). Nos propres observations vont dans le même sens ; elles suggèrent en outre qu'il s'agit d'une particularité associée au degré de pigmentation du duvet en général, et non au seul gène $E$. En d'autres termes, un effet pléiotropique lié à la coloration nous paraît vraisemblable. Il serait intéressant de vérifier si les besoins accrus en riboflavine associés à l'extension du noir d'après BERNIER et COONEY (I954) se retrouvent dans les comparaisons entre génotypes relatifs à d'autres loci d'extension ou d'intensité de la pigmentation.

Pour le gène $K$, notre résultat paraît à première vue contradictoire avec celui de Gredina (citée par HuTr, I949, et par LANDAuer, I967). Cependant, les données de GREDINA sont une comparaison entre des croisements donnant des descendants à emplumement rapide ou lent, et non une comparaison directe de la mortalité embryonnaire de ces deux types à l'intérieur des mêmes familles, ce qui introduit une cause de variabilité supplémentaire ; 1'effectif du croisement donnant la descendance à emplumement rapide est relativement faible pour une comparaison portant sur des pourcentages; enfin, les taux d'éclosion présentés sont, dans l'ensemble, assez bas.

Notre résultat, net en ce qui concerne notre population, demanderait à être vérifié sur d'autres souches. On peut se demander s'il trouverait ou non une explication dans des besoins accrus en certains nutriments (acides aminés soufrés par exemple?) associés à la pousse précoce des plumes chez l'embryon.

Quant au gène $P$, aucun résultat publié, à notre connaissance, n'apporte d'information concernant son effet possible sur la mortalité embryonnaire ou juvénile; le cas est identique pour $P o$ et la mortalité du poussin. Ici aussi, nos observations devraient être étendues dans 1'avenir à d'autres populations. 
Il en est de même du gène $B_{1}$ et de son effet constaté dans nos conditions sur la mortalité des poulettes et des poules en ponte. Il est trop tôt pour affirmer si cet effet est lié à l'allèle $B l$ avec effet de dominance, ou à l'état hétérozygote $B l b l$. Dans ce dernier cas, et si cet effet associé avait un caractère de généralité, on pourrait penser à l'utiliser dans cərtains croisements.

Enfin, il sera intéressant de vérifier la mortalité plus élevée aux divers stades suggéréa par nos données pour les individus $R R$ homozygotes pour le gène " crête en rose ". Ce désavantage sélectif supposé pourrait expliquer l'existence, que nous avions signalée antérieurement dans deux autres populations (MÉRAT, Ig67a), d'un déficit important du génotype $R R$ parmi les femelles à crête en rose prises comme reproductrices et issues d'un croisement du type $\hat{\sigma} R r \times q R r$.

Cette interprétation impliquerait que la différence supposée de mortalité entre génotypes $R R$ et $R r$ ne soit pas limitée à la seule population de Jouy et représente un effet pléiotropique du gène $R$. On connaît déjà la fərtilité inférieura des coqs $R R$ (COCHEZ, I95I ; CRAWFORD et MERRITT, I963). Par contre, d'après une comparaison, sur des effectifs limités, entre zygotes $R R$ et $R r$ de génotype connu d'après leur descendance (MÉRAT, I967 $a$ ) ou entre zygotes de phénotype $(R)$ ou $(r)$ issus du croisement $R r \times R r$ (données non publiées), les performances de croissance, de ponte ou les caractères des œufs ne diffèrent pas de façon décelable entre les génotypes $R R$ et $R r$ dans notre population. Dans une autre souche, un léger retard de maturité sexuelle et une ponte des premiers mois un peu inférieure sont suggérés pour les poules $R R$ (données non publiées).

\section{B. - Différences entre croisements réciproques ou associées aux génotypes parentaux}

La mortalité plus grande en fin d'incubation lorsque les mères sont $C c$ au lieu de $c c$ est à première vue surprenante, car nous avions trouvé antérieurement (MÉ́RAT, I967 b) un taux global d'éclosion supérieur pour le premier génotype maternel comparé au second. Ces résultats étant tous deux très significatifs, il faudrait supposer que les mères colorées $C c$ présentent, chez leurs descendants pendant l'incubation, une répartition de la mortalité par stade différente de celle qui existe lorsque la mère est $c c$. Nous avions d'ailleurs suggéré, concernant la différence globale du taux d'éclosion, la possibilité, par exemple, de fécondations plus espacées des poules $c c$ entraînant à la fois un taux de fertilité et d'éclosion un peu inférieurs.

Quant à la différence entre croisements réciproques pour le gène " extension du noir " pour la mortalité des poulettes après 8 semaines, elle est intéressante par son amplitude relativement grande et mérite d'être vérifiée sur des effectifs plus nombreux et à partir d'autres souches.

D'une façon générale, il resterait à préciser, dans chacun des cas discutés cidessus, si les différences de mortalité observées sont dues ou non à une sensibilité à des maladies particulières.

D'un point de vue pratique, les différences apparues dans la mortalité après 8 semaines seraient les plus importantes, en particulier celles associées au génotype zygotique au locus $B l$ et au génotype maternel au locus $E$, dans la mesure où elles se généraliseraient à d'autres populations. Cependant, des différences de l'ordre de celles observées dans la mortalité embryonnaire ou au stade poussin peuvent avoir 
des conséquences économiques appréciables, répercutées sur de grands effectifs. Nos résultats pourraient poser, par exemple, la question de 1'opportunité d'utiliser le gène $P$ pour son effet favorable à la survie des jeunes dans nos conditions, ou de revoir dans certains cas le choix de la souche maternelle en croisement (rôle du gène $s$ en particulier).

La mortalité globale est typiquement un caractère de faible héritabilité sur lequel la sélection, ordinairement, a peu de prise. Si un ou plusieurs gènes connus ont sur elle un effet favorable ou défavorable, même limité, il peut valoir la peine d'en tenir compte.

Reçu pour publication en octobre 1972.

\section{REMERCIEMENTS}

Nous remercions M. J. Guillaume, Station de Recherches avicoles, I. N. R. A., et M. F.-H. RICARD, Station expérimentale d'Aviculture du Magneraud, I. N. R. A., de leurs utiles suggestions et critiques à la lecture de ce manuscrit.

\section{SUMMMARY}

POSSIBLE RELATIONS BETWEEN EMBRYONIC AND POST-EMBRYONIC MORTALITY RATE AND THE PRESENCE OF KNOWN GENES IN THE FOWL,

Possible associations were looked for between known genes with visible effect (colour or morphology), in families segregating for these genes, and mortality at three stages (embryos at the end of the incubation period, chicks till the age of eight weeks, pullets from eight weeks to Io months). The main results are the following :

- For three colour genes $(C, I, E)$, the more melanized embryos have a higher mortality (from I.5 to 3 p. IOo). This difference is prolonged in the first weeks of the chick's life for the $C$ and perhaps the $I$ gene.

- At the $K$ locus, there appears an excess (+ $3 \mathrm{p}$. roo) of the percentage of dead during the end of the incubation period among fast-vs. slow-feathering zygotes.

- Pea-comb $(P P)$ embryos and chicks have a better livability $(+5$ and +2 p. Ioo respectively at the 2 stages) than single-comb $(p p)$ ones.

- Mortality is about $2 \mathrm{p}$. xoo higher for chicks with five toes than for 4 -toe ones, the reverse seeming to exist before hatching.

- For pullets after 8 weeks of age, the only significant difference concerns the $B l$ locus : heterozygous females for dilution of black $(B l b l)$ have a 5 p. Ioo lower mortality than non-diluted homozygous ones $(b l b l)$.

- At the three stages, an inferior survival rate of rose-comb homozygotes $(R R)$ compared to the other genotypes is suggested, but requires confirmation by additional data.

- Mortality at the end of incubation is higher ( $+3 \mathrm{p}$. 1oo) when the dam is colored $(C c)$ than when it is homozygous for the recessive white gene $(c c)$. On the other hand, chick and possibly embryo mortality is lower when the dam has "gold " plumage $\left(s^{-}\right)$than when it has "silver " plumage $\left(S^{-}\right)$.

After 8 weeks of age, pullets issued from a cross between a heterozygous dam for the $E$ gene (extension of black) and a male lacking this allele seem to have less mortality than those produced by the reciprocal cross. cussed.

The interest of these results, if they can be generalized to other populations, is briefly dis - 


\section{RÉFÉRENCES BIBLIOGRAPHIQUES}

Bernier P. E., Cooney T., I954. Black down color and riboflavin deficiency in embryos of the domestic fowl. Xth. World Poultry Congress, Edinburgh, Section papers, 66-7I.

Brumbaugh J. A., Hollander W. F., rg66. Genetics of buff and related color patterns in the fowl. Poultr. Sci., 45, 45I-457.

Cochez L.-P., I95I. Un facteur d'infertilité, équilibré par la sélection, chez la Wyandotte blanche. C. $R$. $I X^{\mathrm{e}}$ Congrès mondial d'Aviculture, Paris, 1, $8 \mathrm{I}-88$.

Crawford R. D., Merrits E. S., I963. The relationship between rose comb and reproduction in the domestic fowl. Canad. J. Genet. Cytol., 5, 89-95.

Hutr F. B., r949. Genetics of the fowl. Mc Graw Hill Book, New York.

Hutr F. B., I951. Lethal action of the gene for extension of black pigment in the fowl. Genetics, 36, $213-234$.

LANDAUER W., 1967. The hatchability of chicken eggs as influenced by environment and heredity. Monogr. University of Connecticut agric. Exp. Sta.

MÉrat P., I962. Quelques relations entre caractères extérieurs à hérédité simple et productivité. C. $R$. $X I I^{\mathrm{e}}$ Congrès mondial d'Aviculture, Sydney, 1, 7I-76.

MÉrat P., I967a. Contribution à l'étude de la valeur sélective associée à quelques gènes chez la poule domestique. I. Différences quantitatives liées au génotype individuel. Ann. Biol. anim. Bioch. Biophys. 7, 79-104.

MÉRAT P., I $967 b$. Contribution à l'étude de la valeur sélective associée à quelques gènes chez la poule domestique. II. "Effets maternels". Ann. Biol. anim. Bioch. Biophys., 7, 183-203.

MÉrat P., I970. Mendellian genetics and selection for quantitative traits in poultry : results and perspectives. World Poult. Sci. J., 26, 57I-586.

Washburn K. W., Smyth J. R., Jr., rg68. Hematology of an inherited anemia in the domestic fowl. Poult. Sci., 47, I4OI-I4II. 